EPJ Web of Conferences 73, 07013 (2014)

DOI: $10.1051 /$ epjconf/20147307013

(C) Owned by the authors, published by EDP Sciences, 2014

\title{
Lepton flavour universality and conservation tests in Kaon decays at CERN
}

\author{
A. Romano ${ }^{\mathrm{a}, \mathrm{b}}$ \\ School of Physics and Astronomy, University of Birmingham, Edgbaston B15 2TT, UK
}

\begin{abstract}
A test of lepton flavour conservation in kaon rare decays by the NA48/2 experiment at CERN SPS is presented. The improved upper limit on the rate of the lepton number violating decay $K^{ \pm} \rightarrow \pi^{\mp} \mu^{ \pm} \mu^{ \pm}$is established with the data sample collected in 2003-2004. A precision test of lepton flavour universality in kaon rare decays by the successor NA62 experiment at CERN SPS is reported. The helicity suppressed ratio $R_{K}=\Gamma\left(K^{ \pm} \rightarrow e^{ \pm} v\right) / \Gamma\left(K^{ \pm} \rightarrow \mu^{ \pm} v\right)$ is measured with a $0.4 \%$ precision using the data sample collected in 2007-2008 and is consistent with the prediction of the Standard Model. The main goal of the NA62 experiment is to measure the branching ratio of the ultra-rare $K^{+} \rightarrow \pi^{+} v \bar{v}$ decay with $10 \%$ accuracy. This will be achieved by collecting $\sim 10^{13} K^{+}$ decays in the fiducial volume. The NA62 estimated sensitivities to rare and forbidden decays of $K^{+}$and $\pi^{0}$, including lepton flavour and number violating modes, and the NA62 potential to improve on several experimental limits are discussed.
\end{abstract}

\section{Introduction}

In the Standard Model (BM) flavour sector the processes which violate Lepton Flavour (LF) symmetry are extremely suppressed, if we simply consider extensions of the SM including right-handed neutrinos. Larger rates (at tree level amplitude) are predicted in more ambitious extensions of the SM,

\footnotetext{
ae-mail: axr@hep.ph.bham.ac.uk

${ }^{b}$ For the NA48/2 and NA62 Collaborations: F. Ambrosino, A. Antonelli, G. Anzivino, R. Arcidiacono, W. Baldini, S. Balev, J.R. Batley, M. Behler, S. Bifani, C. Biino, A. Bizzeti, B. Bloch-Devaux, G. Bocquet, V. Bolotov, F. Bucci, N. Cabibbo, M. Calvetti, N. Cartiglia, A. Ceccucci, P. Cenci, C. Cerri, C. Cheshkov, J.B. Chèze, M. Clemencic, G. Collazuol, F. Costantini, A. Cotta Ramusino, D. Coward, D. Cundy, A. Dabrowski, G. D’Agostini, P. Dalpiaz, C. Damiani, H. Danielsson, M. De Beer, G. Dellacasa, J. Derré, H. Dibon, D. Di Filippo, L. DiLella, N. Doble, V. Duk, J. Engelfried, K. Eppard, V. Falaleev, R. Fantechi, M. Fidecaro, L. Fiorini, M. Fiorini, T. Fonseca Martin, P.L. Frabetti, A. Fucci, S. Gallorini, L. Gatignon, E. Gersabeck, A. Gianoli, S. Giudici, A. Gonidec, E. Goudzovski, S. Goy Lopez, E. Gushchin, B. Hallgren, M. Hita-Hochgesand, M. Holder, P. Hristov, E. Iacopini, E. Imbergamo, M. Jeitler, G. Kalmus, V. Kekelidze, K. Kleinknecht, V. Kozhuharov, W. Kubischta, V. Kurshetsov, G. Lamanna, C. Lazzeroni, M. Lenti, E. Leonardi, L. Litov, D. Madigozhin, A. Maier, I. Mannelli, F. Marchetto, G. Marel, M. Markytan, P. Marouelli, M. Martini, L. Masetti, P. Massarotti, E. Mazzucato, A. Michetti, I. Mikulec, M. Misheva, N. Molokanova, E. Monnier, U. Moosbrugger, C. Morales Morales, M. Moulson, S. Movchan, D.J. Munday, M. Napolitano, A. Nappi, G. Neuhofer, A. Norton, T. Numao, V. Obraztsov, V. Palladino, M. Patel, M. Pepe, A. Peters, F. Petrucci, M.C. Petrucci, B. Peyaud, R. Piandani, M. Piccini, G. Pierazzini, I. Polenkevich, I. Popov, Yu. Potrebenikov, M. Raggi, B. Renk, F. Retière, P. Riedler, A. Romano, P. Rubin, G. Ruggiero, A. Salamon, G. Saracino, M. Savrié, M. Scarpa, V. Semenov, A. Sergi, M. Serra, M. Shieh, S. Shkarovskiy, M.W. Slater, M. Sozzi, T. Spadaro, S. Stoynev, E. Swallow, M. Szleper, M. Valdata-Nappi, P. Valente, B. Vallage, M. Velasco, M. Veltri, S. Venditti, M. Wache, H. Wahl, A. Walker, R. Wanke, L. Widhalm, A. Winhart, R. Winston, M.D. Wood, S.A. Wotton, O. Yushchenko, A. Zinchenko, M. Ziolkowski.
}

This is an Open Access article distributed under the terms of the Creative Commons Attribution License 4.0, which permits unrestricted use, distribution, and reproduction in any medium, provided the original work is properly cited. 

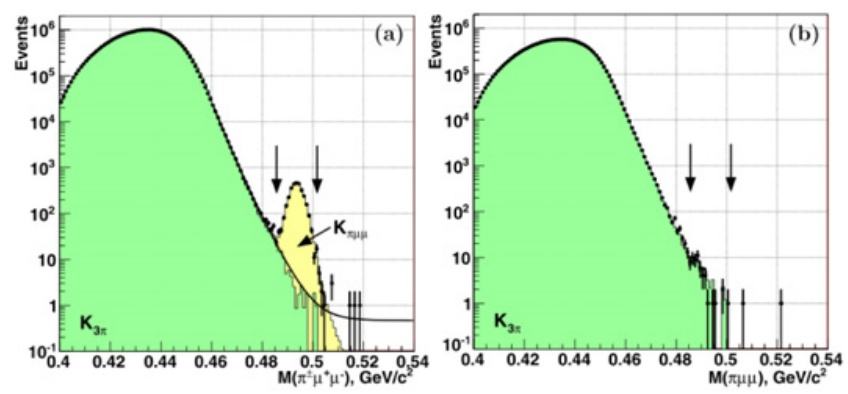

Figure 1. MC and data comparison of the invariant mass distributions for $K^{ \pm} \rightarrow \pi^{ \pm} \mu^{ \pm} \mu^{\mp}$ (left) and $K^{ \pm} \rightarrow$ $\pi^{\mp} \mu^{ \pm} \mu^{ \pm}$(right) decays. Green (yellow) area represents the estimated MC contribution from $K^{ \pm} \rightarrow \pi^{ \pm} \pi^{ \pm} \pi^{\mp}$ $\left(K^{ \pm} \rightarrow \pi^{ \pm} \mu^{ \pm} \mu^{\mp}\right)$ decay. Dots represent the reconstructed data.

including supersymmetry (MSSM) and its modification to include violation of R-parity [1]. Any experimental observation of LF violating processes would provide an unambiguous evidence of new physics (NP). A test of LF universality performed by the NA62 experiment and a test of LF conservation by its predecessor NA48/2, as well as prospects for studies of forbidden LF violating processes within the main research programme of NA62 starting at the end of 2014, are presented in this paper.

\section{Lepton flavour conservation test}

Lepton Number Violation (LNV) in the $K^{ \pm} \rightarrow \pi^{\mp} \mu^{ \pm} \mu^{ \pm}$decay $(\Delta L=2)$ can be generated by the heavy Majorana neutrino exchange, i.e by the same mechanism that leads to the neutrino-less nuclear double $\beta$ decay. The coupling is sensitive to effective $v$ mass (kinematically accessible if $m_{\pi}<m_{v}<m_{K}$ ) and translates into exclusion limits in the space of the parameters of the extended PMNS mixing matrix and the heavy neutrino mass [2]. The process provides the best way to study effects of Majorana neutrinos in the second lepton generation, Until recently, the most stringent limit on the rate of the LNV $K^{ \pm} \rightarrow$ $\pi^{\mp} \mu^{ \pm} \mu^{ \pm}$decay came from a special data set collected in 1997 by the E865 experiment at Brookhaven [3] to measure the rate of $K^{ \pm} \rightarrow \pi^{ \pm} \mu^{ \pm} \mu^{\mp}$. From the study of background to $K^{ \pm} \rightarrow \pi^{ \pm} \mu^{ \pm} \mu^{\mp}$ an upper limit on $B R\left(K^{ \pm} \rightarrow \pi^{\mp} \mu^{ \pm} \mu^{ \pm}\right)<3.0 \times 10^{-9}$ (90\%C.L.) was established.

The NA48/2 experiment at CERN SPS [4] performed the analysis of the same decay channel with a data sample recorded in 2003-2004 with a kaon decay-in-flight technique. Simultaneous $K^{+}$ and $K^{-}$beams, with a central momentum of $60 \mathrm{GeV} / c$ and a narrow momentum band, decayed in a 114 m-long cylindrical vacuum tank housing the fiducial decay volume. The momenta of charged decay products were measured in a magnetic spectrometer and a plastic scintillator hodoscope was used as a fast trigger and for precise time measurements. A Liquid Krypton (LKr) electromagnetic calorimeter, an iron/scintillator hadronic calorimeter and muon detectors were used as vetoes and for particle ID.

The $K^{ \pm} \rightarrow \pi^{ \pm} \mu^{ \pm} \mu^{\mp}$ rate is measured relative to the abundant $K^{ \pm} \rightarrow \pi^{ \pm} \pi^{ \pm} \pi^{\mp}$ normalization channel. The signal and normalization samples were collected concurrently using the same trigger logic. The invariant mass of the three tracks in the $\pi \mu \mu$ hypothesis lies in the range between 485 and $502 \mathrm{MeV} / \mathrm{c}^{2}$ (see Fig. 1); the MC estimation of background gave $52.6 \pm 19.8$ corresponding to the following upper limit [5]: $B R\left(K^{ \pm} \rightarrow \pi^{\mp} \mu^{ \pm} \mu^{ \pm}\right)<1.1 \times 10^{-9}$ (90\%C.L.). Within the near-future NA62 data taking a potential sensitivity of $\sim 10^{-12}$ is reachable due to several improvements, including a higher kaon flux and a better resolution on the invariant mass. 


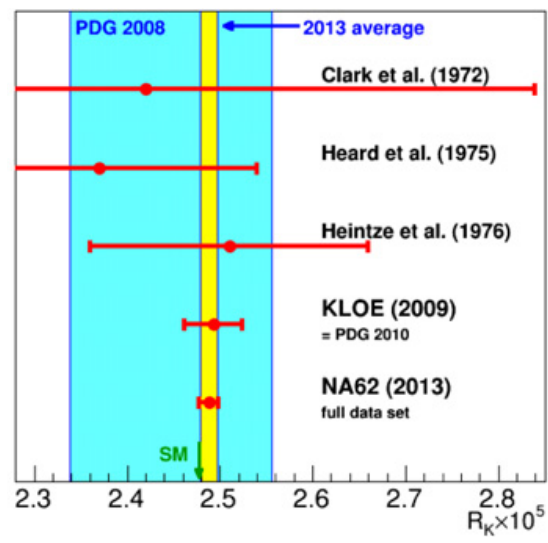

Figure 2. Summary of $R_{K}=\Gamma\left(K_{e 2}\right) / \Gamma\left(K_{\mu 2}\right)$ measurements and SM expectation.

\section{Lepton flavour universality test}

In the $\mathrm{SM}$ the ratio $R_{K}$ of charged kaon leptonic decay rates $\Gamma\left(K^{ \pm} \rightarrow e^{ \pm} v_{e}\right)$ and $\Gamma\left(K^{ \pm} \rightarrow \mu^{ \pm} v_{\mu}\right)$, defined as $R_{K}=\Gamma\left(K_{e 2}\right) / \Gamma\left(K_{\mu 2}\right)$, is predicted to excellent sub-permille precision due to the cancellation of hadronic uncertainties [6] and is sensitive to LFV:

$$
R_{K}(S M)=\left(\frac{m_{e}}{m_{\mu}}\right)^{2} \times\left(\frac{m_{K}^{2}-m_{e}^{2}}{m_{K}^{2}-m_{\mu}^{2}}\right)^{2} \times\left(1+\delta R_{Q E D}\right)=(2.477 \pm 0.001) \times 10^{-5},
$$

where the factor $\left(\frac{m_{e}}{m_{\mu}}\right)^{2} \sim 10^{-5}$ represents the "helicity suppression" term and $\delta R_{Q E D}=(-3.78 \pm$ $0.04) \%$ is a correction term due to the inner bremsstrahlung (IB) radiation included by definition in $R_{K}$ [6]. Beyond the SM the ratio $R_{K}$ is sensitive to minimal super-symmetric (MSSM) and 2 Higgs doublet (2DHM) models, which predict an enhancement of the SM amplitude, at the one-loop level, dominated by LFV terms and mediated by a charged Higgs exchange [7]. The NA62 experiment at CERN SPS performed a measurement of $R_{K}$ at a sub-percent accuracy using the full data sample collected in 2007-2008 with a kaon decay-in-flight technique. The experimental layout for the $R_{K}$ measurement comprised the beam line and detector setup of the earlier NA48/2 experiment; the running conditions were optimized for $K_{e 2}$ data collection. Unseparated secondary beams ( $\left.\pi^{ \pm} / K^{ \pm} \sim 10\right)$, within a narrow momentum band of $74 \mathrm{GeV} / c$ central momentum and $1.4 \mathrm{GeV} / c$ spread (rms), were used. The NA62 data taking strategy for the $R_{K}$ measurement relied on a minimum bias trigger configuration with low kaon beam intensity and it was optimised to measure the two main backgrounds in the $K_{e 2}$ sample: the beam halo muons and the $K_{\mu 2}$ decays with a mis-identified muon.

The total $K_{e 2}$ sample comprises 145,958 candidates with a background contamination of (10.95 \pm $0.27) \%$. The total $K_{\mu 2}$ sample consists of about 43 million candidates with a background contamination of $(0.50 \pm 0.01) \%$ due to the beam halo. The $R_{K}$ result with the full data sample collected by the NA62 collaboration at CERN in 2007-2008 is [8]: $R_{K}=\left(2.488 \pm 0.007_{\text {stat }} \pm 0.007_{\text {syst }}\right) \times 10^{-5}$. The result is consistent with the previous measurements and the SM expectation (see Fig. 2); it is the most precise $R_{K}$ measurement achieved so far and dominates the current world average.

\section{Prospects for LFNV in $K^{+}$and $\pi^{0}$ decays}

The NA62 experiment [9] aims to study the ultra-rare FCNC $K^{+} \rightarrow \pi^{+} \nu \bar{v}$ decay with $K^{+}$decay-inflight technique. The main goal is to measure $B R\left(K^{+} \rightarrow \pi^{+} \nu \bar{\nu}\right)$ with a relative precision of $\sim 10 \%$ in 
Table 1. Lepton flavour and lepton number decay rates of $K^{+}$and $\pi^{0}$ with current experimental upper limits, which can potentially be improved by the NA62 experiment.

\begin{tabular}{lll}
\hline Mode & Physics & UL at 90\% CL (Experiment) \\
\hline$K^{+} \rightarrow \pi^{+} \mu^{+} e^{-}$ & LFV & $1.3 \times 10^{-11}$ (BNL E777/E865) [10] \\
$K^{+} \rightarrow \pi^{+} \mu^{-} e^{+}$ & LFV & $5.2 \times 10^{-10}$ (BNL E865) [3] \\
$K^{+} \rightarrow \pi^{-} \mu^{+} e^{+}$ & LFNV: $\Delta L_{\mu}=\Delta L_{e}=-1$ & $5.0 \times 10^{-10}$ (BNL E865) [3] \\
$K^{+} \rightarrow \pi^{-} e^{+} e^{+}$ & LNV: $\Delta L_{e}=2$ & $6.4 \times 10^{-10}$ (BNL E865) [3] \\
$K^{+} \rightarrow \pi^{-} \mu^{+} \mu^{+}$ & LNV: $\Delta L_{\mu}=2$ & $1.1 \times 10^{9}$ (NA48/2) [5] \\
$K^{+} \rightarrow \mu^{-} v e^{+} e^{+}$ & LFNV: $\Delta L_{e}=2$ & $2.8 \times 10^{-8}$ (Geneva-Saclay) [11] \\
$K^{+} \rightarrow e^{-} v \mu^{+} \mu^{+}$ & LFNV: $\Delta L_{\mu}=2$ & No Data \\
$\pi^{0} \rightarrow \mu^{ \pm} e^{\mp}$ & LFV & $3.6 \times 10^{-10}(\mathrm{KTeV})[12]$ \\
\hline
\end{tabular}

two years of data taking, starting end of 2014. The study of FCNC kaon decay modes is an excellent probe for flavour sector in SM, as well as for indirect search of NP, complementary to B physics. The primary goal of NA62 is to detect $\sim 100 K^{+} \rightarrow \pi^{+} \nu \bar{\nu}$ decays with a ratio signal/background $\sim 10$. Assuming the SM predicted value of $B R\left(K^{+} \rightarrow \pi^{+} v \bar{\nu}\right)=(7.81 \pm 0.80) \times 10^{-11}[13]$ and an acceptance of $\sim 10 \%$, at least $10^{13}$ kaon decays are needed in the fiducial decay region. The high intensity kaon flux, the performances of sub-detectors designed to control backgrounds at the challenging level of $10^{12}$, combined with a flexible trigger system, will allow to perform searches in rare and forbidden kaon and (neutral) pion ${ }^{1}$ decays, including lepton flavour and number violating modes, with an unprecedented precision. By defining the NA62 Single Event Sensitivity (SES) as the inverse of the product between the acceptance and the number of kaon (pion) decays, the estimated NA62 SES on $K^{+}\left(\pi^{0}\right)$ decays is: $\sim 10^{-12}\left(\sim 10^{-11}\right)$. Therefore, referring to Tab. 1, one can argue that the NA62 experiment has the potential to improve on many experimental UL at $90 \% \mathrm{CL}$, up to few order of magnitude, for a wide range of rare and symmetry-violating $\left(K^{+}\right.$and $\left.\pi^{0}\right)$ decays.

\section{References}

[1] L. Littenberg and R. Shrock, Phys. Rev. Lett. 491, 285 (2000)

[2] A. Ali, A. Borisov and N. Zamorin, Eur. Phys. J. C 21, 123 (2001)

[3] R. Appel et al., Phys. Rev. Lett. 85, 2877 (2000)

[4] V. Fanti et al., Nucl. Inst. Meth. A 574, 433 (2007)

[5] J. Batley et al., Phys. Lett. B 697, 107 (2011)

[6] V. Cirigliano and I. Rosell, Phys. Rev. Lett. 99, 231801 (2007)

[7] A. Masiero, P. Paradisi and R. Petronzio, JHEP 0811, 042 (2008)

[8] C. Lazzeroni et al., Phys. Lett. B 719, 326 (2013)

[9] G. Anelli et al., Proposal to measure the rare decay $K^{+} \rightarrow \pi^{+} \nu \bar{v}$ at the CERN SPS (CERNSPSC-2005-013), SPSC-P-326 (2005)

[10] A. Sher et al., Phys. Rev. D 72, 012005 (2005)

[11] A. Diamant-Berger et al., Phys. Lett. B 62, 485 (1976)

[12] E. Abouzaid et al., Phys. Rev. Lett. 100, 2450 (2008)

[13] J. Brod, M. Gorbahn, E. Stamou, Phys. Rev. D 83, 034030 (2011)

\footnotetext{
${ }^{1}$ About $20 \%$ of $K^{+}$undergoes the $K^{+} \rightarrow \pi^{+} \pi^{0}$ decay.
} 